\title{
Karl-Maria Hettlage (1902-1995): un expert au service de l'Europe et des Allemagnes
}

\section{Mauve CARBONELL}

Comment un haut responsable de l'administration du Troisième Reich est-il devenu membre de la Haute Autorité de la Communauté Européenne du Charbon et de l'Acier en 1962? Le parcours de Karl-Maria Hettlage est d'après les biographies officielles assez limpide. Juriste spécialiste des questions financières, universitaire dans différentes facultés de droit allemandes, secrétaire d'Etat aux Finances dans le gouvernement du chancelier Konrad Adenauer à partir de 1958, il est nommé à Luxembourg en 1962 à la suite d'une longue et remarquable carrière administrative et politique.

Cependant le parcours réel de cet homme est loin d'être aussi simple qu'il n'y paraît. On constate qu'après un début de carrière brillant sous la République de Weimar, son ambition le pousse dans les sphères du pouvoir pendant le nazisme. Proche collaborateur d'Albert Speer, il le seconde dans un grand nombre de tâches et assume directement de hautes responsabilités dans les services de l'architecte et ministre d'Adolf Hitler, que ce soit auprès de Speer inspecteur général de la construction pour la transformation de la capitale du Reich ou auprès du ministre de l'Armement dès 1942. Très discret dans l'immédiat après-guerre, il renaît à la vie publique et politique progressivement dans les années cinquante. Comme beaucoup de hauts fonctionnaires, experts ou administrateurs à l'époque nazie, Karl-Maria Hettlage retrouve une place dans la nouvelle Allemagne démocratique. Secrétaire d'Etat aux Finances, il influence la politique financière de son pays. Sa nomination à la Haute Autorité apparaît comme un «accident» dans ce parcours jusque-là national. N'est-il qu'un «technicien», un expert financier au service des administrations et institutions qui lui demandent son concours? Qu'en est-il de sa pensée politique, lui qui sert aussi bien Speer que Adenauer ou la CECA? Et plus particulièrement, qu'en est-il de son travail effectif dans les services de Speer?

Aborder l'histoire de la construction européenne par l'angle biographique peut être particulièrement intéressant. Si les biographies des «grands hommes» de l'histoire européenne (Jean Monnet, Robert Schuman, Alcide De Gasperi, etc.) sont nombreuses, l'approche biographique des élites européennes, fonctionnaires ou dirigeants (membres de la Haute Autorité, commissaires européens, ...) reste à approfondir. ${ }^{1}$ Entreprendre un travail biographique sur les hommes qui ont «fait» l'Europe sans en être les initiateurs connus permet d'élargir les connaissances de l'univers des institutions européennes. Outre les aspects économiques, juridiques et politiques des communautés européennes, la connaissance des hommes qui y ont travaillé et de leurs parcours permet une nouvelle compréhension, un nouveau

1. On peut citer l'une des rares études globales de type biographique sur le sujet, étude déjà ancienne: N. CONDORELLI BRAUN, Commissaires et juges dans les communautés européennes, Librairie générale de droit et de jurisprudence, Paris, 1972. 
regard historique sur les institutions elles-mêmes, sur l'Europe. En effet, qui sont ces hommes qualifiés d' «Européens» parce qu'ils ont travaillé pour une institution européenne? Hettlage, même s'il est un exemple quelque peu extrême, montre par son parcours que les idées préconçues sur l'Européen typique (résistance pendant la guerre, engagement dans les mouvements européens et internationaux, combat pour la démocratie et la paix, ...) sont parfois fausses. L'image véhiculée par l'Europe sur elle-même et sur sa construction s'apparente au mythe, lequel est souvent en contradiction, ou pour le moins en décalage, avec le constat historique. Pour autant, forcer le trait critique aboutirait à l'excès inverse et serait préjudiciable. Le cas de Hettlage ne remet pas en cause le processus profondément démocratique engagé avec la CECA et poursuivi par la construction européenne.

\section{Un début de carrière réussi}

Karl-Maria Hettlage est né le 28 novembre 1902 à Essen, en Rhénanie-du-NordWestphalie. Son père, Karl Hettlage, est marié à Klara Brandenburg et exerce la profession d'avocat. Il occupe dans sa carrière différents postes d'importance politique et administrative (maire de plusieurs villes et préfet $^{2}$ de Münster). ${ }^{3}$ Issu d'une famille bourgeoise, Karl-Maria Hettlage part, à la fin de ses études secondaires, étudier le droit à l'université de Cologne, puis à l'université de Münster. Il commence en 1925 sa carrière scientifique et universitaire à l'Institut de droit international de Kiel comme assistant de Theodor Niemeyer. Ce dernier en fait le secrétaire de la Société allemande de droit international. ${ }^{4} \mathrm{Si}$ l'on ne retrouve presque aucune trace d'engagement politique de jeunesse, on constate cependant son appartenance, pendant trois ans entre 1922 et 1925, au «Freikorps Westfalen». ${ }^{5}$ Il s'en explique dans les années cinquante en déclarant que le corps franc de Westphalie était une formation étudiante émanant de l'Etat prussien à l'université de Münster dans le but d'organiser le combat politique contre les communistes, pendant les «troubles» ${ }^{6}$ dans la région de la Ruhr.

En 1928, il devient assistant à la faculté de droit de l'université de Cologne, en droit public. A cette époque il termine sa thèse de doctorat, qui est publiée dans une revue de droit international. Sa thèse porte sur «L'intervention dans l'histoire du droit international et dans l'enseignement du droit international». ${ }^{7}$ Parallèlement,

2. Regierungsvizepräsident.

3. Bundesarchiv Koblenz (BAK), N1314/20 et entretien avec Peter Hettlage, fils de Karl-Maria Hettlage, le 24 avril 2004.

4. Deutsche Gesellschaft für Völkerrecht.

5. Bundesarchiv Berlin (BAB)/(BDC)6400016976-550, SS-Akte.

Les Freikorps sont des organisations paramilitaires créées au lendemain de la Première Guerre mondiale. Ils regroupent des volontaires monarchistes et ultraconservateurs pour combattre la démocratie, la République, les sociaux-démocrates et les communistes.

6. Landesarchiv Berlin/BRep.031-02.01/Nr11536. Par troubles, Hettlage entend l'occupation française de la Ruhr.

7. Die Intervention in der Geschichte der Völkerrechtswissenschaft und dem System der Völkerrechtslehre, in: Niemeyers Zeitschrift für Internationales Recht, Band 37, 1927. 
entre 1925 et 1929, il est régulièrement Regierungsreferendar (stagiaire du gouvernement) dans les institutions publiques à Cologne et Aix-la-Chapelle. En 1929, il épouse Margarete Brenken, avec qui il a quatre enfants (Peter, Jan Bernt, Birgitta et Karin).

En 1930, il débute réellement sa carrière politique et professionnelle. Il est recruté comme 'juge gouvernemental' dans l'administration de Cologne. Cette même année, il obtient son habilitation à enseigner grâce à un travail sur «la compensation des finances et des charges en tant que problème de droit constitutionnel d'après la Constitution de Weimar». ${ }^{8}$ Il devient ainsi professeur de droit public à l'université de Cologne, à l'âge de 28 ans. Il le reste jusqu'en 1935.

Les circonstances l'amènent par ailleurs à la politique locale. Le président du Deutscher Städtetag (assemblée des délégués des villes allemandes ou congrès des maires allemands) lui demande en 1931 d'effectuer un remplacement au poste d'adjoint aux finances de l'Assemblée. Il exerce ces fonctions pendant quatre ans. Ses diplômes universitaires rapidement obtenus, ses différents postes dans l'administration régionale, ses nombreuses publications dans le domaine fiscal et financier s'ajoutent à un mandat de député du Zentrum au Preussischer Landtag (parlement régional). Il est élu en 1932 dans la circonscription de Cologne-Aix-la-Chapelle. A 29 ans, il est le plus jeune député de son époque.

En 1935, il a déjà une carrière politique, universitaire et professionnelle enviable. Cette année-là, il quitte Cologne pour la capitale du Reich. Il est nommé receveur municipal (Stadtkämmerer) ${ }^{9}$ et entre au conseil municipal de Berlin.

«La fonction de receveur municipal de Berlin, capitale du Reich, fut le premier

sommet professionnel de ma vie. J'entrais dans cette fonction à l'âge de 32 ans et y

restais presque cinq ans. Mon travail m'a fasciné. J'avais réussi». ${ }^{10}$

Les autorités policières nazies valident cette nomination, malgré quelques tergiversations entre l'administration et la police à propos de la «pureté» politique du nouveau nommé. Son appartenance au Zentrum avant 1933 rend notamment méfiant le Reichsleiter ${ }^{11}$ Karl Fiehler. ${ }^{12}$

\section{Nazi d'apparence?}

Rares sont les textes et documents retraçant avec exactitude et précision la vie de Karl-Maria Hettlage entre 1935 et 1945. Les questions qui se posent à la lecture de la suite de sa carrière sont pourtant nombreuses. En effet, comment poursuivre

8. Finanz- und Lastenausgleich als verfassungsrechtliches Problem nach der Weimarer Reichsverfassung.

9. Cf. Landesarchiv Berlin, A Pr. Br. Rep. 060 Nr. 112.

10. Archives privées Peter Hettlage, Mémoires de Karl-Maria Hettlage. Les citations originales en langue allemande ont été traduites par l'auteur.

11. Cadre supérieur politique et administratif du NSDAP.

12. Landesarchiv Berlin, BRep031-02-01/Nr11536. 
une ascension professionnelle, à des postes importants dans l'administration municipale et nationale, en restant en dehors de tout contact avec le pouvoir national-socialiste?

Les faits doivent être lus avec précaution. Son appartenance avérée à la SS ne suffit pas pour faire de lui ni un membre actif de ce corps ni un partisan nazi. Son numéro de SS est le 276909. Il est qualifié SS-Untersturmführer (sous-lieutenant) le 13 septembre 1936, SS-Obersturmführer (lieutenant) un an plus tard et SS-Hauptsturmführer (capitaine) en septembre $1938 .{ }^{13}$ Il s'agit d'un rang d'honneur. Une appartenance à la SS, même inactive et honorifique, n'est pas anodine. Cependant, dans ce cas précis, elle ne semble justifiée que par la nécessité pour un haut responsable municipal d'appartenir, au moins formellement, à une organisation nazie. Après la guerre, chaque fois que Hettlage sera amené à occuper des fonctions importantes, cette appartenance lui posera problème; il lui faudra se justifier. Il le fait une première fois en 1959, alors que Franz Etzel, ministre des Finances, le prend comme sous-secrétaire d'Etat aux Finances. Adenauer demande alors à Hettlage de justifier son rang d'honneur dans la SS. Etzel rapporte les propos de ce dernier au chancelier dans une lettre du 12 mars 1959:

«Le rang d'honneur me fut décerné, en tant que receveur municipal de Berlin, sous l'impulsion du maire. J'étais le seul conseiller municipal berlinois à ne pas appartenir au parti nazi. Malgré des invitations répétées, j'ai toujours refusé. [...] Les avancements aux grades d'Obersturmführer et de Hauptsturmführer ont suivi automatiquement en 1937 et 1938. J'ai peu fait usage de ce rang d'honneur là où l'uniforme était de rigueur». ${ }^{14}$

La suite de la carrière professionnelle de Hettlage engendre un certain nombre de questions. Tout comme l'appartenance d'honneur à la SS, son parcours entre 1939 et 1945 est rarement précisé dans les biographies officielles. S'il fut effectivement licencié de son poste de receveur municipal à Berlin pour raisons politiques, cela ne l'empêcha pas de retrouver rapidement un poste dans l'administration allemande, et à un niveau plus élevé. On le retrouve ainsi dès le printemps 1939, et jusqu'à la fin de la guerre, au service d'Albert Speer ${ }^{15}$ pour la transformation de la capitale du Reich tout d'abord, puis en 1942 au ministère de l'Armement et des Munitions du Reich (qui devient en 1943 le ministère de l'Armement et de la production de guerre). Hettlage connaît Speer du temps où il était membre du conseil municipal de Berlin. En effet, le travail de Speer en tant que Generalbauinspekteur (GBI) für die Reichshauptstadt Berlin (inspecteur général de la Construction pour la capitale du Reich) ${ }^{16}$ l'amène naturellement à

13. BAB/(BDC)6400016976-550, SS-Akte.

14. BAK, N1314/441.

15. Sur la personne Albert Speer, cf. J. FEST, Albert Speer, Perrin, Paris, 2001; A. SPEER, Au cœur du Troisième Reich, Fayard, Paris, 1971; Idem., Journal de Spandau, Robert Laffont, Paris, 1975. $\mathrm{Au}$ sujet de son ministère, cf. notamment G. JANSSEN, Das Ministerium Speer - Deutschlands Rüstung im Krieg, Verlag Ullstein, Berlin/Frankfurt am Main, 1968.

16. Albert Speer fut nommé par Hitler à ce poste en 1937 et chargé de transformer Berlin selon les desseins du Führer. Par la suite, on nommera pour plus de facilité l'ensemble des services de Speer par l'abréviation GBI. 
rencontrer et à travailler avec les administrateurs municipaux. On remarque la présence de Hettlage aux côtés de Speer et d'un certain nombre de ses collaborateurs à un déjeuner donné par Hitler, le 14 juin 1938. Karl-Maria Hettlage est encore receveur communal.

«Le Führer salue ses invités: Speer, Esser, Goebbels, Dorpmüller, Todt, Lippert, Ohnesorge, Schwerin, Steeg, Kleinmann, Hanke, Langer, Pückel, Rheinhardt, Zörner, Gutterer, Hettlage, Engel, Elkart, Jeserich, Leibrandt, Fiehler, Ley, Wolters, Stephan, Röttcher». ${ }^{17}$

Hettlage écrit:

«J'avais déjà eu des contacts personnels avec Albert Speer alors que j'occupais mes fonctions de receveur municipal. [...] Speer cherchait en moi un conseiller financier». ${ }^{18}$

Il intègre par ailleurs à cette époque le comité directeur de la Commerzbank et conserve cette fonction, qui sera en quelque sorte sa fonction «officielle» pendant toute la durée de la guerre.

Quand Hettlage quitte l'administration municipale, il rejoint Speer dont il devient le représentant (Vertreter) à diverses occasions, comme lors de réceptions officielles. Ainsi, le 22 septembre 1941:

«En l'honneur des invités allemands, le ministre plénipotentiaire allemand donna une réception, le ministre de l'éducation hongrois un petit-déjeuner et le maire de la ville de Budapest un goûter. L'inspecteur général de la construction, représenté par le Prof. Hettlage, donna un petit-déjeuner en l'honneur d'architectes hongrois à l'Institut scientifique allemand». ${ }^{19}$

Mais Speer lui confie surtout le service des finances au sein du GBI. Il participe à la réorganisation des services et s'occupe plus particulièrement des relations avec la municipalité de Berlin, qu'il vient de quitter et qu'il connaît bien. Le GBI est un service très structuré, ${ }^{20}$ divisés en bureaux, eux-mêmes subdivisés en sections. Hettlage est le chef du bureau central «Administration et économie» ${ }^{21}$ en 1941-1942. Ce bureau s'occupe principalement des aspects financiers et juridiques des activités du GBI.

L'un des aspects du travail du GBI est détaillé dans l'ouvrage de Susanne Willems sur l'expropriation des Juifs de Berlin. ${ }^{22}$ Elle montre comment Speer et

17. BAK, N1340/27, compte-rendu du déjeuner donné par Hitler le 18 juin 1938 pour la pose de la première pierre de la Maison du Tourisme de Berlin et de seize autres constructions entrant dans le projet de transformation de Berlin.

18. Mémoires de Karl-Maria Hettlage, op.cit.

19. BAK, N1318/1, Chronique Wolters, Jan-Dez. 1941, p.75.

20. H.C. LÖHR, Intelligenz und Macht - Über das Organisationstalent Albert Speer als Generalbauinspektor für die Reichshauptstadt Berlin, in: Berlin in Geschichte und Gegenwart, Jahrbuch des Landesarchives Berlin, Berlin, 1995, pp.171-183.

21. Hauptamt II des GBI, Verwaltung und Wirtschaft.

22. S. WILLEMS, Der entsiedelte Jude - Albert Speers Wohnungsmarktpolitik für den Berliner Hauptstadtbau, Edition Hentrich, Berlin, 2000. 
ses services ont organisé l'expulsion des Juifs de la capitale et on apprend dans quelles mesures Hettlage y a contribué.

«Speer et Hettlage ont décidé de faire des Juifs de Berlin des sans-logis et de les concentrer, dans des conditions misérables, dans un ghetto invisible. [...] Cette politique développée par Speer - de son propre pouvoir et de sa propre initiative, sans intimations d'autres parties -, tournée vers ses propres buts, plaça "l'autorité de réorganisation" [Neugestaltungsbehörde] sous la responsabilité de Hettlage à partir de janvier 1941». ${ }^{23}$

Karl-Maria Hettlage devient l'un des véritables seconds d'Albert Speer. Il s'occupe plus particulièrement des aspects administratifs, financiers et organisationnels de cette politique d'expropriation. Suzanne Willems cite ainsi le compte-rendu d'une réunion ayant eu lieu le 28 janvier 1941 entre les représentants du GBI, de la police, de la municipalité de Berlin, de la Gauleitung, etc.

«L'évacuation des Juifs devait être menée à terme policièrement. Hettlage demanda lors de la réunion du 28 janvier 1941: "la SS est-elle en mesure d'évacuer 100 logements en 14 jours? Au total, environ 250 logements juifs doivent être reloués, et donc évacués, d'ici le 28 février de cette année. La SS possède une liste des logements juifs concernés par l'évacuation"». ${ }^{24}$

Ces logements «évacués» sont ensuite reloués à la propre clientèle de Speer. Ce travail main dans la main entre les services de Speer et la SS conduit à la ghettoïsation des Juifs de Berlin, dont le sort sera par la suite celui des Juifs d'Europe: déportation et extermination. Qu'en est-il de l'implication de Hettlage dans cette politique? Responsable avec les services de Speer de l'expropriation et de l'expulsion des Juifs berlinois, c'est indéniable. Si Albert Speer en est l'initiateur, Hettlage semble être l'un de ses exécutants.

Au début de 1942, après la mort de Fritz Todt, Hitler nomme Speer ministre de l'Armement et des Munitions du Reich. ${ }^{25}$ Son équipe de collaborateurs, dont Hettlage, le suit dans ses nouvelles fonctions. On trouve, relaté avec beaucoup de précisions, l'activité des services de Speer dans les chroniques de Rudolf Wolters. Ce dernier, un proche collaborateur de Speer au GBI, puis au ministère de l'Armement, a tenu un journal des services entre 1941 et 1945.

«A la fin de l'année 1940, je suggérai à Speer de consigner dans une 'Chronique' mensuelle tous les évènements importants ayant eu lieu dans ses différents services. Je commençai mon activité de chroniqueur le $1^{\text {er }}$ janvier 1941». ${ }^{26}$

Comme au sein du GBI, Speer place Hettlage à la tête des services financiers du ministère de l'Armement. Celui-ci est divisé en quatre sections principales: ${ }^{27}$ Economie et finances (Hettlage); personnel et administration (Haasemann); droit et organisation (Schattenmann); culture (Hoffmann). Les fonctions de Hettlage sont

23. S. WILLEMS, op.cit., p.161.

24. BAK, R120/Nr.1975., cité dans S. WILLEMS, op.cit., p. 196.

25. G. JANSSEN, op.cit.

26. BAK, N1318/58.

27. Le ministère sera réorganisé début 1944 mais les fonctions de Hettlage resteront sensiblement les mêmes. 
multiples: représentant de Speer, chef de bureau du GBI, chef des services financiers et économiques du ministère de l'Armement. Les questions financières en temps de guerre sont particulièrement importantes. C'est ainsi que par exemple, il analyse dans une réunion confidentielle certains problèmes économico-financiers concernant l'augmentation de la production d'armes en 1942.

«A la question de savoir comment il serait possible d'augmenter encore la production d'armes aux vues de la situation des matières premières, le Prof. Hettlage répondit que cela pouvait être atteint par différents moyens». ${ }^{28}$

Il détaille ensuite les solutions économiques pouvant être apportées à cette question, compte tenu de l'évolution de la guerre.

\section{Le Bureau de l'Armement}

Karl-Maria Hettlage occupe d'autres fonctions au sein de l'administration Speer. Le 13 juillet 1942, il se voit confier la direction du Bureau de l'Armement (Rüstungskontor). Ce bureau s'occupe des approvisionnements en marchandises spécifiques et en matières premières, tout d'abord en Allemagne, puis dans les régions occupées. Comme l'écrit Rudolf Wolters dans sa chronique de juillet 1942:

«Afin d'exécuter certaines tâches économiques spéciales, un bureau de l'Armement fut créé, qui entre autres avait les fonctions suivantes: service de compensation du métal, service d'indemnisations et de déductions dans le cas de stocks excessifs et opération de récupération; opportunités économiques et financières pour le renforcement des câbles de cuivre par l'utilisation de matériel et de machines inutilisés; financement de constructions et orientation du secteur de l'armement, ... etc. A la présidence du Conseil du Bureau de l'Armement le ministre nomma le Professeur Hettlage». ${ }^{29}$

Il s'agit là d'une société d'Etat, gérée par le ministère de l'Armement et de la Production de guerre.

Il est intéressant aussi de voir ce que cette société a réellement engendré dans le système national-socialiste pendant la guerre. Le 21 septembre 1943 se sont réunis, sous la direction de Hettlage, des représentants de différents ministères, de la SS, de l'armée et de l'industrie. Le but de cette réunion est de donner une forme juridique à une nouvelle société, la Mittelwerk $\mathrm{GmbH}$ placée sous la tutelle et le contrôle du Bureau de l'Armement de Hettlage. Elle est dotée d'un capital de départ d'un million de Reichsmark et son siège doit s'installer à Berlin. Mais le lieu principal d'activité de la Mittelwerk se situe plus au sud, à côté de Nordhausen. La Mittelwerk est en effet responsable de la production des fusées A4, plus connues sous le nom de V2, ainsi que de la production de V1. Au début de la guerre, elles sont en partie produites à Peenemünde, mais elles sont rapidement déplacées à côté

28. BAK, NS19/2062.

29. BAK, N1318/2 Jan-Dez 1942, p. 69. 
de Nordhausen, dans les montagnes du Harz, à cause des bombardements alliés sur les sites de production. La main d'œuvre employée dans les ateliers vient de Buchenwald, et pour faciliter l'organisation du «travail», un camp est construit sur le site de production, le camp de Dora, ou Mittelbau-Dora ${ }^{30}$ dirigé par le chef de brigade SS Hans Kammler.

Le ministère Speer, même s'il entre souvent en conflit avec la SS à propos des prérogatives de chacun (en particulier sur la question de la main d'œuvre), collabore avec les services de Heinrich Himmler. C'est ainsi que le commandement sur le terrain du camp de Dora est aux mains de la SS, alors que les services administratifs compétents assurant la gestion de la production des fusées (la Mittelwerk) émanent du ministère de l'Armement. Sur Hans Kammler, Speer écrit ceci:

«Himmler, en s'attachant Kammler, avait fait un choix significatif. [...] Au printemps 1942, il avait nommé cet homme, qui avait été jusqu'à cette date un haut fonctionnaire responsable des constructions au ministère de l'Air, directeur du service chargé des constructions de la SS et, à l'été 1943, il l'affecta au programme de construction des fusées. Au cours de la collaboration qui s'ensuivit, le nouvel homme de confiance de Himmler se révéla un calculateur froid et brutal, un fanatique [...]. A l'époque, l'objectivité froide de Kammler me plaisait: de nombreuses tâches à exécuter en firent mon partenaire, sa position présumée mon concurrent $[\ldots] »{ }^{31}$

L'assemblage des pièces des fusées par les détenus des camps de concentration est souterrain, les conditions sanitaires et de travail ressemblent à celles de beaucoup d'autres usines employant les détenus des différents camps de concentration en Allemagne et dans les pays occupés. Entre novembre 1943 et avril 1945, date de la libération du camp, on dénombre plus de 20.000 travailleurs forcés ayant péri à Dora. Par ailleurs, plus de 13.000 fusées ont été produites. Hettlage ne peut ignorer ce qu'il dirige indirectement. Mais les buts et objectifs de l'économie de guerre, de la production d'armes doivent être atteints. Chef du service financier et économique du ministère de l'Armement, il œuvre avec les moyens qui lui sont donnés - ils sont nombreux - pour atteindre ces objectifs. L'organisation de la production de V2 grâce aux travailleurs de camps de concentration est un exemple de moyens dont l'administration Speer dispose pour permettre la poursuite de la guerre. Hettlage est l'un des rouages - importants - du système aux ramifications multiples mis en place par le régime nazi pour atteindre ses buts guerriers et politiques.

Hettlage se situe donc pendant les dernières années de la guerre au sommet de la pyramide administrative dans le domaine de l'armement. Il est un organisateur influent du secteur. Sa lettre de juillet 1944 à Speer montre à quel point il est impliqué dans l'organisation du secteur de l'armement:

30. Cf. A. SELLIER, Histoire du camp de Dora, La Découverte, Paris, 2001;

M. BORNEMANN, Geheimprojekt Mittelbau: vom zentralen Öllager des Deutschen Reiches zur grössten Raketenfabrik im Zweiten Weltkrieg, Bernard und Graefe, München, 1994.

31. A. SPEER, op.cit., p.526. 
«Je considère comme indispensable de poursuivre le projet de décret du Führer sur l'exportation d'armes. Projet discuté avec le ministre de l'Economie, qui donne son accord. L'OKW ${ }^{32}$ veut apparemment échapper au débat. Conseil de réponse à Keitel [lettre de Keitel à Speer du 30 juin 1944]: réorganisation de l'exportation d'armes avec le concours nécessaire du ministère de l'Economie. [...] L'OKW n'est plus responsable de l'exportation d'armes, il n'est plus que le gardien des aspects militaires. La Centrale d'exportations de l'industrie de l'armement (AGK), qui jusqu'à présent dépendait de l'OKW, doit dépendre du ministère de l'Economie. Le rendement de cette communauté d'exportation de matériel de guerre doit être augmenté. Jusqu'à présent, je n'ai pris que des contacts sans engagement avec les représentants de l'industrie de l'armement au sujet de la réorganisation industrielle». ${ }^{33}$

Au début de l'année 1943, en plus de ses nombreuses fonctions, Speer lui offre la direction de la Commission sur le commerce des armes. A la date du 30 janvier 1943, au rang des promotions, Rudolf Wolters note ceci:

«L'insigne d'or honoraire du Parti national-socialiste fut décerné à Saur par le Führer, le titre de professeur fut décerné au Dr. Müller de l'entreprise Krupp sur proposition du Ministre, également par le Führer. Le professeur Hettlage fut nommé Wehrwirtschaftsführer (chef de l'économie militaire)». ${ }^{34}$

Speer évoque l'importance de son collaborateur dans une lettre qu'il lui adresse pour son quarante-deuxième anniversaire, en 1944:

«Je vous remercie en même temps pour les services que vous m'avez rendus et que vous avez rendus à l'Armement allemand en tant que responsable des finances et de l'économie. Je formule le vœu que, dans l'année qui vient, vous puissiez mener à bien votre travail - pour lequel les responsabilités sont grandes - avec la même force d'action et la même santé. [...] Heil Hitler!». ${ }^{35}$

\section{La fidélité au chef et à la «famille Speer»}

Speer et ses collaborateurs forment un groupe très soudé, une sorte de famille. Les liens qui unissent ces hommes travaillant ensemble jusqu'en 1945 perdurent longtemps après la fin de la guerre. La caricature faite par l'un des proches collaborateurs de Speer en $1941,{ }^{36}$ dont le titre est: «Le monastère de l'auto restriction - ou la vie dévote de la confrérie Speer», ${ }^{37}$ est à ce sujet significative. Elle provient d'un petit fonds de documents laissés par W. Schelkes, qui travaillait avec Speer en tant que chef de service au GBI. W. Schelkes explique, dans un commentaire, le contenu de la caricature:

32. Oberkommando der Wehrmacht.

33. BAB/R3/1582/Bd12, pp.197 sqq., Lettre de Hettlage à Speer du 7 juillet 1944.

34. BAK, N1318/3 Jan-Juni 1943, p.12.

35. BAB/R3/1582/Bd12, p.200, lettre de Speer à Hettlage du 24 novembre 1944.

36. BAK, Kleine Erwerbung 864, Schelkes, ${ }^{\circ} 25$.

37. «Das Kloster zur Selbstbeschränkung - oder das gottselige Leben der Bruderschaft "vom Speere"» 
«En 1941, Speer voulait se retirer de ses nombreuses activités - qui s'étendaient sur tout le Reich. Mon collègue Stephan caricatura cette situation en dessin: Speer sur la colonne dans les nuages, devant lui ses plus étroits collaborateurs: au premier plan le Dr. Wolters se délectant du calme; à droite Stephan faisant un rapport sur Berlin; à gauche Schelkes - le jardinier - arrosant quelques fleurs. Dans les arcades de la cour du monastère on relève: Behrendt (administration), Dr. Fränk (questions juridiques), Prof. Hettlage (service des réalisations), Prof. Brugmann, Bohr, Clahes et Nagel». ${ }^{38}$

On retrouve la «confrérie» dans les chroniques de Rudolf Wolters. Par exemple, lors du décès de l'un des collaborateurs (Brugmann) de Speer, au printemps 1944:

«Au soir de ce jour, Speer avait invité sur son bateau de Werbelinsee ses plus anciens collaborateurs: Bohr, Clahes, Fränk, Hettlage, Liebel, Schelkes, Stephan et Wolters, afin d'évoquer ensemble la mémoire de notre Brugmann, à la veille de l'inhumation. Le soir tombait quand le chef est arrivé. Sur le pont du bateau il évoqua devant ses collaborateurs réunis la mort de son ami Brugmann avec des mots sincères et humains». ${ }^{39}$

Cet aspect «famille» au sein du ministère, on le retrouve plus tard, pendant les années qu'Albert Speer passe en prison ${ }^{40}$ puis après sa libération en 1966. En effet, les anciens collaborateurs de l'architecte de Hitler et ministre de l'Armement et de la Production de guerre lui sont restés fidèles. Ils l'ont défendu, ils se sont occupés de ses affaires quand il était en prison, ils l'ont soutenu. Par exemple, en 1952, Hettlage et Wolters sont chargés par leur ancien maître de s'occuper de certaines de ses affaires. Dans une lettre de Speer à Hettlage, datée du 13 novembre 1952, on peut lire ceci:

«Notre ami R. [Rudolf Wolters] m'a écrit que vous n'avez toujours pas perdu l'envie de m'aider dans mes affaires d'héritage. Si vous vouliez vous tenir éloigné de ce champs de vaches désagréable, je vous supplierais sincèrement de ne pas perdre patience. Vous savoir à mes côtés m'apaise grandement». ${ }^{41}$

Après la libération de Speer, Hettlage se rend régulièrement chez son chef d'autrefois. ${ }^{42}$ Quand ce dernier lui envoie ses mémoires, son ancien collaborateur lui répond:

«Si je vous remercie aujourd'hui par cette lettre pour l'envoi amical de vos mémoires, c'est parce que j'aurais voulu avoir lu ce livre auparavant. J'ai rarement lu un livre qui me touchait si personnellement. Beaucoup de passages m'ont refait vivre ma propre vie, passé déjà disparu. C'est particulièrement vrai pour le chapitre sur la "Transformation de la capitale du Reich Berlin" et pour la dernière année au ministère de l'Armement. D'autres chapitres rendent compte d'événements totalement nouveaux pour moi. Je me suis étonné de votre mémoire exceptionnelle pour les caractères, les évènements et les dates». ${ }^{43}$

38. BAK, Kleine Erwerbung 864, Schelkes, $n^{\circ} 25$.

39. BAK, N1318/5 Jan-Juni 1944, 31 mai 1944, p.110.

40. Speer fut condamné à 20 ans de prison par le tribunal de Nuremberg; il les passa à la prison de Spandau.

41. BAK, N1340/179.

42. BAK, N1340/27.

43. BAK, N1340/27, lettre de Hettlage à Speer, 21 octobre 1969. 
Peter Hettlage, fils de Karl-Maria Hettlage témoigne:

«C'était en quelques sorte une amitié. Le fait est que mon père et Speer se sont rencontrés à nouveau quand Speer fut libéré. Quand on fêtait quelque chose d'important (les 70 ans de mon père ou les noces d'or de mes parents), Speer était là». 44

Wolters et Hettlage continuent également de correspondre après la guerre. Ils se connaissent bien, échangent des propos au sujet des affaires d'Albert Speer. Wolters écrit même un poème pour les 65 ans de Hettlage, un mélange entre ironie et sérieux, entre sous-entendus et lyrisme:

«Comme tu atteins tes soixante-cinq ans / avec des fanfares retentissantes, / en passant par l'Institut de recherches économiques, les banques et la CECA / On te souhaite quelques vœux de bonheur / On parle déjà de ton grand parcours dans la vie, / de quelques grands épisodes, / et des réussites de tes ambitions. / On te tresse des couronnes, on t'envoie des odes. / Et les trous dans ton parcours / se fondent avec clémence dans le brouillard / Car Wiesenthal et ses cuisiniers / tiennent leur flèche empoisonnée». 45

Ce que Hettlage pensait vraiment du régime qui le gouvernait est difficile à savoir. On ne peut ici se référer qu'aux rares écrits ou lettres évoquant cette période que l'on retrouve de lui. Dans une lettre de Speer déjà citée, après avoir lu les mémoires de son ancien chef, il écrit ceci:

«Vous me dédiez votre livre "en souvenir de temps lointains, mes remerciements pour beaucoup". Ces temps lointains me sont, et à vous aussi certainement, toujours moins imaginables. De plus en plus souvent je me souviens de quels excès terribles était capable cette dictature, dans les circonstances de l'époque. Je conçois trop bien que la génération de nos enfants ne veuille tout simplement pas le croire. [...] Je me pose moi-même souvent la question de savoir comment j'ai pu, en tant qu'être humain intelligent et au caractère sérieux, surmonter ces années». 46

Dans une autre lettre à Speer, en réaction à la publication du Journal de Spandau, ${ }^{47}$ Hettlage écrit:

«D'un autre côté, c'est toujours une chose désagréable d'exposer ses propres pensées, ses propres sentiments, devant une foule ignorante qui ne comprend pas et qui en général n'éprouve que de la curiosité; une foule pour qui beaucoup de noms de cette période invraisemblable ne signifie plus rien». ${ }^{48}$

Tout ce qu'il est possible de connaître sur la vie de Hettlage nous montre à quel point l'image donnée de lui dans les biographies officielles est erronée. A titre d'exemple, on trouve dans un article nécrologique sur lui, concernant la période avant 1945:

«Les conflits avec les forces nationales-socialistes étaient inévitables. Ils conduisirent Hettlage à être licencié du poste de conseiller municipal et à son

44. Entretien avec Peter Hettlage.

45. BAK, N1340/27, Poème de Rudolf Wolters, 27 novembre 1967.

46. BAK, N1340/27, lettre de Hettlage à Speer, 21 octobre 1969.

47. A. SPEER, Journal de Spandau, op.cit.

48. BAK, N1340/27, lettre de Hettlage à Speer, 21 septembre 1975. 
changement d'orientation vers l'économie privée. De 1938 à 1951, il appartient au comité directeur de la Commerzbank, mais il fut cependant appelé pendant la guerre à des devoirs administratifs dans le domaine de l'économie et des finances». ${ }^{49}$

Cette phrase discrète évoque donc d'une manière furtive son activité au ministère de Speer. Il est dit ici que Hettlage fut obligé par l'état de guerre d'effectuer ce service. On peut également lire cette affirmation dans un curriculum vitæ datant de 1950 et en rapport avec sa nomination à l'université de Mayence: «1942-1944: Service militaire au service financier du ministère de la production». ${ }^{50} \mathrm{Ce}$ C.V. semble avoir été écrit par Hettlage lui-même, probablement pour les services administratifs de l'université. Dans la plupart des notices biographiques, on lit qu'il s'est contenté pendant la guerre de ses fonctions dans le secteur privé (Commerzbank). S'il est effectivement employé par la Commerzbank, ${ }^{51}$ qui constitue pourtant sa principale source de revenu, Hettlage passe beaucoup plus de temps au service de Speer qu'au service de la banque.

Comment interpréter ce parcours sous le régime nazi? Est-il un homme engagé au service d'un régime et d'une idéologie ou un technicien, expert financier au service d'une administration et d'un chef? N'est-il qu'un administrateur très compétent, apolitique, accomplissant son travail quelles que soient les circonstances? On peut se dire qu'il est difficile de faire un travail comme le sien pendant la guerre sans avoir conscience des circonstances, sans connaître les conséquences de certains actes et de certaines décisions, sans avoir de jugements sur les gouvernants qu'il côtoie, qui lui donnent des ordres, qui lui demandent de faire des rapports, de prendre des décisions. Il est un acteur direct du régime: sa participation effective à certaines affaires le démontre (expropriation des Juifs de Berlin, activités du Bureau de l'Armement et de la Mittelwerk). Ses responsabilités dans les évènements décrits jusque-là sont indiscutables.

\section{De l'administration Speer au ministère des Finances de la RFA}

Pendant la dernière année de la guerre, Karl-Maria Hettlage se trouve confronté à la police. La Gestapo l'arrête à deux reprises dans des circonstances peu claires. La première confrontation a lieu après l'attentat raté contre Hitler le 20 juillet 1944. La Gestapo le suspecte d'avoir des liens avec le complot et l'arrête. Elle le relâche immédiatement. («Mon arrestation fut automatiquement décidée parce que j'avais été député du Zentrum avant 1933»). ${ }^{52}$

Hettlage évoque également des liens avec Goerdeler et les sympathisants du complot; liens non connus de la Gestapo. Dans les derniers mois de la guerre, les

49. Archives privées de Peter Hettlage, article biographique, Nachruf, Karl-Maria Hettlage 1902-1995.

50. BAK, N1314/20.

51. Les archives de la Commerzbank ne sont pas accessibles.

52. Mémoires de Karl-Maria Hettlage, op.cit. 
soupçons à son encontre refont surface et il est obligé de fuir en janvier 1945, sur le conseil de Speer, pour éviter une arrestation et les conséquences imprévisibles qu'elle peut avoir. Il fuit à la campagne, trouve refuge dans un premier temps chez l'un de ses amis. En mars, il se cache à Hambourg où il reste jusqu'à la fin de la guerre.

Après la guerre, il est arrêté. Pour les alliés, il est important en tant que témoin. D'où son emprisonnement à Badnauheim, au château de Kransberg (en Hesse). Les alliés veulent savoir comment l'Allemagne a pu mener la guerre (financièrement notamment), comment fonctionnait l'administration Speer. C'est ainsi que les services de l'armée américaine l'interrogent longuement. Il en sort notamment un rapport de huit pages sur lui: «Report on the examination of Karl Hettlage, head of the economic and financial division of the Speer ministry». ${ }^{53} \mathrm{Ce}$ rapport passe en revue une partie des fonctions et actions de Hettlage auprès de Speer, ainsi que ses propres propos sur son rôle et sur le fonctionnement du ministère:

«Il [Hettlage] revendique le fait de ne pas avoir été un membre du parti nazi. Catholique, il était opposé à l'idéologie nazie. Il a dû quitter l'administration municipale berlinoise parce qu'il était considéré par Goebbels comme peu fiable. [...] Hettlage, en tant que receveur municipal, a dû s'opposer aux plans extravagants de Speer. Néanmoins, ce conflit n'a apparemment pas empêché Speer de l'engager en tant que conseiller financier en 1941».54

Dans ce document, on peut lire l'histoire vue par Hettlage, qui tente de se rendre le moins important possible aux yeux des Américains. Il est parfois difficile de faire la juste part des choses, de distinguer les omissions, les erreurs, les dissimulations. Quoi qu'il en soit, il fut libéré rapidement par les Américains.

Par la suite, la justice allemande s'intéresse à lui dans le cadre du processus de dénazification. ${ }^{55} \mathrm{Il}$ est obligé de s'expliquer longuement, en particulier sur son rang dans la SS. Il donne bien sûr dans les documents contenus dans le dossier de dénazification sa propre version de l'histoire et est disculpé en 1948 par la commission de dénazification. Malgré cette décision de 1948, la justice lui demandera régulièrement de fournir des explications sur son passé. Cependant, aux vues de ses fonctions effectives dans les services de Speer, il aurait pu être bien plus inquiété. Son manque de «visibilité» au sein du régime et de l'administration nazie constitue un avantage sérieux. Il ne fait en effet pas partie de la «scène intérieure» nazie, il ne s'affiche pas en public, le grand public ne le connaît pas. A l'inverse de beaucoup d'autres qui cherchent la reconnaissance et la gloire à travers des fonctions dans le parti, la SS, les ministères, l'administration, etc.

«Hettlage, un collaborateur de Speer, plutôt calme, extrêmement compétent, n'atteignit dans les temps qui suivirent [les années de guerre] pas vraiment les feux de la rampe de la reconnaissance publique que les autres désiraient ardemment».56

53. Archives nationales américaines, document numéro NND775058, copie conservée dans les archives privées de Peter Hettlage, «Rapport sur Karl Maria Hettlage, chef de la division économique et financière du ministère Speer».

54. Ibid., p.1.

55. Landesarchiv Berlin/ B.REP031-02-01/Nr.11536, Dossier de dénazification.

56. G. JANSSEN, op.cit., p.41. 
Le fait de ne pas être connu et reconnu comme acteur du régime lui permet de réintégrer progressivement la vie publique après-guerre.

Pendant les années qui suivent la fin de la guerre, Karl-Maria Hettlage cantonne ses activités au secteur privé et à la Commerzbank. Il habite à ce moment-là à Hambourg. Par ailleurs, il a la volonté, dans ces années d'après-guerre, de se faire une place dans le monde universitaire allemand. Il était déjà professeur de droit public à l'université de Cologne avant la guerre. Sa carrière universitaire s'était interrompue avec sa nomination au conseil municipal de Berlin, en 1935. En 1951, il obtient une chaire de professeur à l'université de Mayence. Il l'obtient notamment grâce à ami, Erich Welter, professeur à l'université de Mayence (fondateur de l'Institut de recherche en politique économique en 1950 de cette même université et co-fondateur de la Frankfurter Allgemeine Zeitung), comme le montrent les échanges de lettres entre les deux hommes. ${ }^{57}$ Welter lui écrit le 19 décembre 1950:

«Elle [lettre de Hettlage] vous a entre-temps largement rendu service. Je vous fais part confidentiellement du fait que l'université a décidé à l'unanimité de vous placer en tête de liste pour la succession du professeur Schätzel. [...] Seul le caractère particulier de notre relation m'incite à vous confier ce secret». ${ }^{58}$

Dans une notice écrite par Welter lui-même, en rapport avec l'article paru en l'honneur des 70 ans de Hettlage, ${ }^{59}$ on peut lire ceci:

«Il était à l'époque nazie receveur municipal de Berlin, ensuite conseiller financier autant que je sache - dans l'équipe de Speer. Après la guerre, il est arrivé, par un chemin que je ne connais pas (et sur lequel je ne sais précisément pas s'il fut avec ou sans Nuremberg), au conseil d'administration de la Commerzbank. Il fut nommé à Mayence sur ma proposition». ${ }^{60}$

Plus tard, alors qu'il évoque ses souvenirs avec nostalgie, Hettlage lui répond:

«J'ai eu beaucoup de chance dans la vie et au moins la moitié des grands moments que j'ai vécus, je les dois à un hasard providentiel. L'un de ces hasards providentiels fut aussi le jour où notre rencontre pendant la guerre vous conduisit à Hambourg, où je travaillais à la Commerzbank, pour me proposer une chaire à l'université de Mayence». ${ }^{61}$

Hettlage retrouve ainsi une place dans la vie publique allemande par le biais de l'université. Expert financier reconnu pour ses compétences, il intéresse les dirigeants politiques allemands. Il se rapproche de la CDU et rencontre Franz Etzel. Ce dernier, vice-président de la Haute Autorité de la CECA à partir de 1952, est rappelé à Bonn par Adenauer en 1957. Il est nommé ministre des Finances. Etzel fait alors appel à Hettlage et lui confie la direction du budget. La décision est entérinée à l'unanimité par le conseil des ministres du 17 décembre 1957. Il occupe tout d'abord le poste de directeur du budget en 1958, puis il est nommé en 1959

57. BAK, N1314/20, Fonds Erich Welter.

58. BAK, N1314/20.

59. Cf. Frankfurter Allgemeine Zeitung, du 28 novembre 1972, n²76, p.14.

60. BAK, N1314/198, notice de Welter sur Hettlage, 28 novembre 1972.

61. BAK, N1314/145, lettre de Hettlage à Welter, 24 janvier 1973. 
secrétaire d'Etat aux Finances. Hettlage, dont l'avenir politique semblait compromis à la fin de la guerre, est un exemple parmi d'autres de la reconversion des élites administratives du régime nazi. Cette reconversion de certaines élites est pointée du doigt, de façon peu sérieuse et caricaturale, par les autorités est-allemandes. Hettlage est ainsi «dénoncé» par l'ouvrage publié par la propagande de la RDA sur «les criminels de guerre nazis en RFA et à Berlin-Ouest». ${ }^{62}$ Les historiens allemands se sont penchés sur la question de la reconversion des élites nazies, qu'elles soient administratives, culturelles, juridiques, intellectuelles ou économiques. On peut ainsi évoquer l'ouvrage collectif sur le sujet dont la rédaction est dirigée par Wilfried Loth et Bernd Rusinek. ${ }^{63}$ Après la période de dénazification intense de l'immédiat après-guerre (condamnations, internements), les années cinquante, dans le contexte du début de la guerre froide, offrent une amnistie générale pour beaucoup de condamnés.

Il est intéressant de voir que les nouvelles fonctions de Hettlage le conduisent à s'occuper, notamment, du dossier du dédommagement des victimes de la guerre, lui qui travaillait au sein du ministère qui permit à l'Allemagne de prolonger la guerre grâce à l'efficace organisation de la production! Il répond ainsi, dans une lettre adressée au député Ferdinand Friedenburg, au sujet des essais médicaux réalisés sur des êtres humains:

«Comme tous les persécutés ayant subi des sévices corporels où dont la santé fut altérée, les victimes d'expériences médicales ont également droit à un dédommagement, d'après la loi fédérale de dédommagement. Il consiste en un capital de dédommagement ou une rente permanente. Pour les cas particuliers qui n'entrent pas dans le cadre de la loi, l'arrêt du gouvernement du 26 juillet 1951 prévoit une aide sociale pour les survivants d'expériences médicales. Dans le cadre de cette action, 1413 demandes ont été réglées jusqu'à aujourd'hui, dans 490 cas une aide spéciale a été accordée, 551 demandes ont dû être refusées. Jusqu'à 25.000 Marks par individu ont été payés dans le cadre de l'aide spéciale. Au total, 3,2 millions de Marks d'aide ont été payés sur la base de l'arrêt du gouvernement de $1951 »{ }^{64}$

Hettlage se sent dans son élément au ministère des Finances dirigé par Etzel. «C'était le but de sa vie professionnelle: secrétaire d'Etat aux Finances». ${ }^{65}$ Etzel est souvent absent à cause de ses problèmes de santé et Hettlage prend de l'importance au sein du ministère. Il dit lui-même avoir pu mener sa propre politique financière.

62. Nationalrat der nationalen Front des demokratischen Deutschland Dokumentationszentrum der staatlichen Archivverwaltung der DDR, Braunbuch, Kriegs- und Naziverbrecher in der Bundesrepublik und in Westberlin, Staatsverlag der DDR, Berlin, 1968.

63. Sur les élites «reconverties» du régime nazi, cf. W. LOTH, B. RUSINEK (Hrsg.), Verwandlungspolitik. NS-Eliten in der westdeutschen Nachkriegsgesellschaft, Campus Verlag, Frankfurt/New-York, 1998; U. HERBERT, Rückkehr in die Bürgerlichkeit? NS-Eliten in der Bundesrrepublik, in: B. WEISBROD (Hrsg.), Rechtsradikalismus in der politischen Kultur der Nachkriegszeit. Die verzögerte Normalisierung in Niedersachsen, Hahn, Hannover, 1995, pp.157-173.

64. BAK, N1114/30a, Fonds Ferdinand Friedensburg, lettre de Hettlage à Friedensburg.

65. Entretien avec Peter Hettlage. 
Mais cette situation ne dure pas. Etzel démissionne en 1961. Son successeur est le libéral Heinz Starke du FDP. Les relations entre le nouveau ministre et son secrétaire d'Etat sont pour le moins délicates. Hettlage note: «De caractère, il était exactement le contraire de Franz Etzel: nerveux, incertain, méfiant et jaloux. [...] En octobre 1962, j' ai dû aviser le chancelier Adenauer qu'un travail profitable entre le ministre et son secrétaire d'Etat n'était plus possible». ${ }^{66}$ Le conflit ne peut pas durer. Konrad Adenauer

«annonça soudainement à son cabinet sa décision de nommer le secrétaire à Luxembourg, bien qu'il ait promis ce poste aux syndicats. L'intéressé n'accueillit pas de bon gré cette nomination qui paraissait une mesure d'ostracisme. Mais il était vain qu'il s'oppose à son ministre et au chancelier: il accepta donc». ${ }^{67}$

\section{La Haute Autorité sans convictions?}

Partir pour la Haute Autorité de la CECA n'entre vraiment pas dans les projets et les ambitions de Hettlage.

«Starke n'a été ministre des Finances qu'une petite année. J'avais jeté l'éponge deux semaines trop tôt. Le Conseil des Ministres de la CECA me nomma membre de la Haute Autorité à Luxembourg le 14 octobre 1962». ${ }^{68}$

On ne peut pas dire que ce soit son engagement européen qui le pousse à la Haute Autorité. Contrairement à la plupart des autres membres du collège, on ne trouve pas trace chez lui d'une pensée européenne, voire même d'un embryon d'intérêt particulier pour l'Europe. Il débarque à Luxembourg sans l'avoir voulu et ce, dans des circonstances qui ne sont pas habituelles non plus. Le scandale éclate dix jours après sa nomination. La presse révèle alors son appartenance à la SS avant guerre. Tous les journaux européens se font l'écho de la nouvelle. Par exemple, dans la dernière semaine d'octobre 1962, le journal belge Le Peuple publie plusieurs articles consacrés à l'affaire:

«Un ex-SS à la tête de la CECA? [...] "La voix internationale de la résistance" nous informe que d'après ses archives, un Dr. Karl Hettlage, né le 28 novembre 1902, aurait adhéré en 1936 à la SS sous le matricule 276.909 pour être affecté, à partir de 1938, à l'état-major du quartier général de la SS où il fut nommé capitaine». ${ }^{69}$

Les informations données sont assez superficielles et exagérées, mais le scandale est là. Hettlage et le gouvernement allemand réagissent mollement en déclarant qu'il ne s'agissait que d'une appartenance d'honneur à la SS et qu'un tribunal de dénazification l'acquitta en 1948. Le Peuple rapporte ces réactions tout en continuant à se demander:

66. Mémoires de Karl-Maria Hettlage, op.cit.

67. N. CONDORELLI BRAUN, op.cit., p.84.

68. Mémoires de Karl-Maria Hettlage, op.cit.

69. Le Peuple, 26.10.1962, n²57, p.1. 
«Bonn veut maintenir le Dr. Hettlage à la CECA. La Haute Autorité va-t-elle prendre ses responsabilités? [...] Que M. Hettlage, doté d'une belle pension, cultive ses jardins à Bonn. C'est l'affaire des Allemands. Mais sa place n'est pas à la tête d'une institution supranationale, appelée à s'occuper directement des affaires intérieures de pays démocratiques». ${ }^{70}$

De son côté la Haute Autorité garde le silence. Les choses en restent là. L'affaire s'estompe et Hettlage s'installe à Luxembourg. Dans l'ouvrage de référence sur l'histoire de la Haute Autorité, il n'est pas fait référence à son passé: ${ }^{71}$

«Les gouvernements des Etats membres nomment Karl Maria Hettlage, venu du ministère fédéral des Finances. Sa compétence en matière financière, l'appui d'Etzel qui l'a fait venir au ministère à Bonn dès 1958, le soutien des milieux industriels allemands permettent à cet ancien haut fonctionnaire et professeur d'Université d'assumer avantageusement les tâches confiées jusque-là à Potthoff à la présidence du groupe de travail Investissements et Finances». ${ }^{72}$

Il est difficile d'imaginer que Dirk Spierenburg, qui collabore à cet ouvrage n'ait pas été au courant de cette affaire puisqu'il était en poste à Luxembourg au moment de la nomination de Karl-Maria Hettlage. Sa nomination porte un coup à l'image de la Haute Autorité, qui décline par ailleurs pour d'autres raisons (démissions et départs de membres, perspectives de fusion des exécutifs des trois communautés). La Haute Autorité n'est en rien responsable. C'est le gouvernement allemand qui a pris cette décision. Hormis la connaissance de son appartenance honorifique à la $\mathrm{SS}$, que sait-on réellement sur son passé à ce moment-là? Que savent les autres membres de la Haute Autorité? Et même, que sait le gouvernement allemand? On sait qu'Adenauer demande des explications à Hettlage, par l'intermédiaire de Franz Etzel, mais uniquement sur son grade SS. Cette appartenance à la SS est en quelque sorte le point le plus visible de son passé, celui qui marque le plus les esprits. Son travail avec Albert Speer semble quant à lui être inconnu.

Il reste cinq ans à la Haute Autorité; cinq années durant lesquelles il n'est pas en première ligne. Il est là aussi loin d'être le plus «visible». Il met au service de la communauté européenne ses compétences, son expertise financière, juridique et économique. Il le fait tout comme il l'a fait pour le ministère des Finances à Bonn, pour les administrations d'Albert Speer à Berlin. Il semble que de ces trois postes importants qu'il occupe durant sa carrière, son passage à Luxembourg soit celui qui le ravisse le moins. En 1967, date de la fusion des exécutifs des trois communautés, Hettlage a 65 ans. Il quitte la Haute Autorité et est rappelé par Bonn. Il reprend le poste de secrétaire d'Etat aux Finances. Comme si la Haute Autorité n'avait été qu'une parenthèse, il réintègre les fonctions qu'il occupait avant d'être nommé à Luxembourg. Cette fois, c'est Franz Joseph Strauss (CSU), ministre des Finances de la «grande coalition», qui le fait venir. Deux ans plus tard, en 1969, une nouvelle

70. Le Peuple, 27-28.10.1962, n²58, p.2.

71. R. POIDEVIN, D. SPIERENBURG, Histoire de la Haute Autorité de la Communauté Européenne du Charbon et de l'Acier - Une expérience supranationale, Bruylant, Bruxelles, 1993.

72. Ibid., pp.633-634. 
coalition sociale-libérale prend la relève. Hettlage, qui n'a pas de mandat politique, disparaît alors du ministère des Finances en même temps que les ministres CDU de l'ancienne grande coalition. Par ailleurs, il a déjà atteint la limite d'âge pour un secrétaire d'Etat. Et même si les dérogations sont nombreuses, à 67 ans, l'âge de la retraite approche d'autant plus que les perspectives politiques s'annoncent peu favorables pour un membre de la CDU non-élu. Comme il le dit lui-même à Albert Speer en 1969:

«De mon côté, je termine en ce moment ma mission d'après-guerre en tant que secrétaire d'Etat au ministère des Finances, en partie à cause de la limite d'âge, en partie à cause du changement politique». ${ }^{73}$

Ce court passage au ministère des Finances marque la fin de la carrière politique et publique de Hettlage. Après 1969, il commence une vie de retraité actif. C'est à l'Institut für Wirtschaftsforschung (IFO) de Munich qu'il consacre le plus de temps. Il en est le président depuis 1965 et le reste jusqu'en 1976. Cet institut de recherche économique a été fondé en 1949, notamment par Ludwig Erhard. Il est l'un des plus importants d'Allemagne et regroupe des entreprises, chercheurs universitaires et experts en économie qui travaillent par exemple sur la conjoncture économique, les politiques sociales, économiques et financières. L'IFO propose ensuite des mesures, des réformes au gouvernement allemand ou des études aux entreprises et à de nombreuses institutions nationales et internationales. Il est également membre de plusieurs comités directeurs de fondations, telle la Fritz Thyssen Stiftung. Karl-Maria Hettlage meurt le 3 septembre 1995 à l'âge de 92 ans.

\section{Conclusion}

L'image qui est donnée de Karl-Maria Hettlage est celle d'un technicien peu politisé. Il est un administrateur efficace et compétent. Le travail auprès d'Albert Speer apparaît comme le plus important dans sa carrière. L'homme et l'administration qu'il sert sont fortement marqués idéologiquement; les actions menées ne peuvent pas lui être inconnues puisqu'il y participe activement. «Responsable mais pas coupable»? Il fut jugé par un tribunal de dénazification et acquitté. Ce passé silencieux, qui resurgit avec force et de façon inexacte au cours de sa vie, ne l'a pas empêché de continuer après guerre une carrière universitaire, administrative et politique. La République Fédérale, en réintégrant nombre d'anciens fonctionnaires du régime nazi, leur offre une seconde vie. Hettlage va même jusqu'à servir l'Europe, en exerçant là aussi ses compétences. Il la sert sans exprimer un enthousiasme particulier pour l'idée européenne. La première communauté européenne et les hommes qui ont participé à sa mise en place souffrent du décalage entre une réalité historique indéniable et l'image véhiculée

73. BAK, N1340/27, lettre de Hettlage à Speer, 21 octobre 1969. 
d'une histoire politiquement correcte. Le parcours de Hettlage montre ce décalage. Il n'est en rien l'«Européen» typique décrit dans les biographies officielles. Cependant, il est important de signaler à quel point son parcours est singulier. $\mathrm{Ce}$ parcours qui aboutit à l'Europe en 1959 est un exemple extrême et exceptionnel pour les institutions européennes. Il n'est bien sûr pas représentatif des hommes de la Haute Autorité en particulier, et des communautés européennes en général. De même, aucun des parcours de l'un de ces hommes - membres de la Haute Autorité, fonctionnaires européens etc. - ne peut être pris comme modèle pour l'élaboration d'une vie d'Européen typique. Dans sa singularité extrême, Karl-Maria Hettlage montre aussi les particularités de chacun, de la vie de chacun de ceux qui avant et après lui occupèrent les mêmes fonctions. 
